\title{
Three-body bound states in atomic mixtures with resonant p-wave interaction
}

\author{
Maxim A. Efremov, ${ }^{1,2}$ Lev Plimak, ${ }^{1,3}$ Misha Yu. Ivanov, ${ }^{3}$ and Wolfgang P. Schleich ${ }^{1}$ \\ ${ }^{1}$ Institut für Quantenphysik and Center for Integrated Quantum Science and Technology (IQ $\left.{ }^{S T}\right)$, \\ Universität Ulm, 89081 Ulm, Germany \\ ${ }^{2}$ A.M. Prokhorov General Physics Institute, \\ Russian Academy of Sciences, 119991 Moscow, Russia \\ ${ }^{3}$ Max-Born-Institut, 12489 Berlin, Germany
}

\begin{abstract}
We employ the Born-Oppenheimer approximation to find the effective potential in a three-body system consisting of a light particle and two heavy ones when the heavy-light short-range interaction potential has a resonance corresponding to a non-zero orbital angular momentum. In the case of an exact resonance in the $p$-wave scattering amplitude, the effective potential is attractive and longrange, namely it decreases as the third power of the inter-atomic distance. Moreover, we show that the range and power of the potential, as well as the number of bound states are determined by the mass ratio of the particles and the parameters of the heavy-light short-range potential.
\end{abstract}

Introduction. One of the most intriguing phenomenon of few-body physics is the Efimov effect [1], which manifests itself in an infinite number of weakly bound threebody states if at least two of the three two-body subsystems exhibit a single weakly $s$-wave bound state or resonance. The underlying effective potential is attractive and decreases as the second power of the inter-atomic distance [1]. In this Letter, we consider a three-body system consisting of a light particle and two heavy ones, when the heavy-light short-range interaction potential has a weakly bound or quasi-bound, i.e., resonant state with a non-zero orbital angular momentum. We show that in the case of the exact $p$-wave resonance, the effective potential is also attractive and of long-range, but now decreases as the third power of the inter-atomic distance [2].

The Efimov effect occurs in systems where the twobody scattering length $a_{0}$ is large compared to the characteristic radius $R_{0}$ of the two-body interaction and displays an universal behavior, that is the details of the underlying short-range physics become irrelevant. Examples of systems with a large scattering length are halo nuclei [3, 4] and the helium trimer [5]. In both cases $a_{0}$ is exceedingly large, but not tunable. However, in order to observe the Efimov spectrum, it is crucial to be able to tune $a_{0}$. In the domain of ultracold atomic gases this task is achieved by Feshbach resonances [6] and different features of the three-body recombination process as well as the scattering of the atom off the shallow dimer, which are associated with the Efimov effect, have been measured [7] in this way. An additional prerequisite to detect many Efimov states is the use of an atomic mixture [8] with heavy atoms of mass $M$ and light ones of mass $m$, since in this case the ratio of two neighboring bound-state energies is $\left|E_{n+1} / E_{n}\right| \simeq 1$ for $M / m \rightarrow \infty$ [9 11].

In our Letter we consider such an atomic mixture. However, in contrast to the standard Efimov scenario we focus on an exact $p$-wave resonance in the heavy-light short-range potential and determine the effective inter- action potential between the two heavy atoms. Moreover, we demonstrate that the spectrum of bound states is solely determined by the mass ratio of the heavy and light particles, and the width of the $p$-wave state.

Experimentally, such a mixture with a $p$-wave resonance has already been realized with $\mathrm{K}$ and $\mathrm{Rb}$ [12], as well as with $\mathrm{Li}$ and $\mathrm{Rb}[13$, corresponding to the mass ratios $m_{\mathrm{K}} / M_{\mathrm{Rb}} \approx 0.5$ and $m_{\mathrm{Li}} / M_{\mathrm{Rb}} \approx 0.1$. Therefore, both mixtures are promising candidates to verify our predictions.

The Efimov effect is intimately connected to the absence [9] of any characteristic length scale. Since for a $p$-wave resonant interaction there is a natural non-zero effective range, there cannot [14, 15] be an Efimov effect in this case. Nevertheless, we find a series of three-body bound states. This surprising fact is a consequence of this non-zero effective range.

Born-Oppenheimer approach. Our three-body system consists of a light particle which interacts with two heavy particles and therefore can be easily analyzed within the Born-Oppenheimer approximation [16]. For this reason, the Schrödinger equation for the full wave function $\Phi(\mathbf{r}, \mathbf{R})=\Psi(\mathbf{r} ; \mathbf{R}) \chi(\mathbf{R})$ separates into two equations and the one for the light particle reads

$$
\left[-\frac{\hbar^{2}}{2 \mu} \Delta_{\mathbf{r}}+U\left(\mathbf{r}_{-}\right)+U\left(\mathbf{r}_{+}\right)\right] \Psi(\mathbf{r} ; \mathbf{R})=-\frac{\hbar^{2} \kappa^{2}}{2 \mu} \Psi(\mathbf{r} ; \mathbf{R})
$$

with $\mathbf{r}_{ \pm} \equiv \mathbf{r} \pm \frac{1}{2} \mathbf{R}$. Here $\mu \equiv 2 m M /(2 M+m) \approx m$ and $\mathbf{R}$ denote the reduced mass and the separation between the two heavy particles, respectively. For the sake of simplicity we assume the potential $U$ to be spherically symmetric, i.e., $U(\mathbf{r})=U(r)$, and to have the finite range $R_{0}$, i.e., $U\left(r>R_{0}\right)=0$.

The bound-state energies

$$
\mathcal{V}(\mathbf{R}) \equiv-\frac{[\hbar \kappa(\mathbf{R})]^{2}}{2 \mu}
$$

of the light particle, corresponding to different expressions for $\kappa$ following from Eq. (1), serve as effective in- 
teraction potentials for the relative motion of the heavy particles given by

$$
\left\{\Delta_{\mathbf{R}}+\frac{M}{\hbar^{2}}\left[E-\mathcal{V}(\mathbf{R})-V_{0}(\mathbf{R})\right]\right\} \chi(\mathbf{R})=0,
$$

where $E$ and $V_{0}$ are the total three-body energy and the direct heavy-heavy interaction potential [10], respectively.

For atoms $V_{0}$ is typically a short-range potential, and has for large distances a van-der-Waals tail, $V_{0} \sim 1 / R^{6}$. We now show that in the case of an exact $p$-wave resonance, the effective potential $\mathcal{V}$ is a long-range one, $\mathcal{V} \sim 1 / R^{3}$, and therefore $V_{0}$ has no effect on the behavior of the total potential for large distances.

Interaction potential from scattering approach. Next we determine $\mathcal{V}$ by a self-consistent scattering of the light particle off the two heavy ones [2]. For this purpose, we cast Eq. (1) into the integral equation [16]

$$
\Psi(\mathbf{r})=-\frac{\mu}{2 \pi \hbar^{2}} \int d \mathbf{r}^{\prime}\left[U\left(\mathbf{r}_{-}^{\prime}\right)+U\left(\mathbf{r}_{+}^{\prime}\right)\right] \Psi\left(\mathbf{r}^{\prime}\right) \frac{e^{-\kappa\left|\mathbf{r}-\mathbf{r}^{\prime}\right|}}{\left|\mathbf{r}-\mathbf{r}^{\prime}\right|} .
$$

Since the total heavy-light potential $U\left(\mathbf{r}_{-}\right)+U\left(\mathbf{r}_{+}\right)$is nonzero only inside two spheres of radius $R_{0}$ centered at $\mathbf{r}= \pm \frac{1}{2} \mathbf{R}$, we represent Eq. (44) as the superposition

$$
\Psi(\mathbf{r})=\Psi^{(-)}(\mathbf{r})+\Psi^{(+)}(\mathbf{r})
$$

of the two waves

$$
\Psi^{( \pm)}(\mathbf{r}) \equiv \int_{\left|\mathbf{r}^{\prime} \pm \frac{\mathbf{R}}{2}\right| \leq R_{0}} d \mathbf{r}^{\prime} \sigma^{( \pm)}\left(\mathbf{r}^{\prime}\right) \frac{e^{-\kappa\left|\mathbf{r}-\mathbf{r}^{\prime}\right|}}{\left|\mathbf{r}-\mathbf{r}^{\prime}\right|}
$$

with

$$
\sigma^{( \pm)}(\mathbf{r}) \equiv-\frac{\mu}{2 \pi \hbar^{2}} U\left(\mathbf{r} \pm \frac{1}{2} \mathbf{R}\right) \Psi(\mathbf{r})
$$

The expansion

$$
\frac{e^{-\kappa\left|\mathbf{r}-\mathbf{r}^{\prime}\right|}}{\kappa\left|\mathbf{r}-\mathbf{r}^{\prime}\right|}=8 \sum_{l=0}^{\infty} \sum_{\left|m_{l}\right| \leq l} \mathcal{I}_{l}\left(\kappa r^{\prime}\right) \mathcal{K}_{l}(\kappa r) Y_{l m_{l}}\left(\mathbf{n}_{\mathbf{r}^{\prime}}\right) Y_{l m_{l}}\left(\mathbf{n}_{\mathbf{r}}\right)
$$

into the modified spherical Bessel functions $\mathcal{I}_{l}(z) \equiv$ $\sqrt{\pi /(2 z)} I_{l+1 / 2}(z)$ and $\mathcal{K}_{l}(z) \equiv \sqrt{\pi /(2 z)} K_{l+1 / 2}(z)$ [17], which is valid for $r>r^{\prime}$, transforms Eq. (6) into

$$
\Psi^{( \pm)}(\mathbf{r})=\sum_{l=0}^{\infty} \sum_{\left|m_{l}\right| \leq l} C_{l m_{l}}^{( \pm)} \mathcal{K}_{l}\left(\kappa r_{ \pm}\right) Y_{l m_{l}}\left(\mathbf{n}_{\mathbf{r}_{ \pm}}\right)
$$

Here $Y_{l m_{l}}\left(\mathbf{n}_{\mathbf{r}}\right) \equiv Y_{l m_{l}}\left(\theta_{r}, \varphi_{r}\right)$ are the spherical harmonics with $\mathbf{n}_{\mathbf{r}} \equiv \mathbf{r} / r=\left(\theta_{\mathbf{r}}, \varphi_{\mathbf{r}}\right)$.

We regard the coefficients $C_{l m_{l}}^{( \pm)}$determined by the integral in Eq. (6) as independent variables and apply scattering theory to obtain from Eq. (9) explicit equations for $C_{l m_{l}}^{( \pm)}$coupled by the $S$-matrix elements of the potential $U$ [2]. For this purpose we consider a vicinity of the first potential well, that is $\mathbf{r}=-\frac{1}{2} \mathbf{R}+\mathbf{x}$ with $|\mathbf{x}| \approx R_{0}$, where the total solution

$$
\Psi\left(-\frac{\mathbf{R}}{2}+\mathbf{x}\right)=\sum_{l=0}^{\infty} \sum_{\left|m_{l}\right| \leq l} R_{l m_{l}}(\kappa|\mathbf{x}|) Y_{l m_{l}}\left(\mathbf{n}_{\mathbf{x}}\right)
$$

given by Eq. (5) can be expanded into the spherical harmonics. Here the radial wave function

$$
\begin{aligned}
R_{l m_{l}}(\kappa|\mathbf{x}|)= & C_{l m_{l}}^{(+)} \mathcal{K}_{l}(\kappa|\mathbf{x}|)+ \\
& \pi(-1)^{l} \mathcal{I}_{l}(\kappa|\mathbf{x}|) \sum_{l^{\prime}=0}^{\infty} \mathfrak{A}_{l l^{\prime}}^{\left(m_{l}\right)} C_{l^{\prime} m_{l}}^{(-)}
\end{aligned}
$$

is determined by the sum of the two contributions resulting from $\Psi^{( \pm)}\left(-\frac{1}{2} \mathbf{R}+\mathbf{x}\right)$ defined by Eq. (9), and the coefficients

$$
\begin{aligned}
\mathfrak{A}_{l l^{\prime}}^{\left(m_{l}\right)}(\kappa R) \equiv & \frac{1}{\pi} \sqrt{\frac{2 l+1}{2 l^{\prime}+1}} \sum_{L=0}^{\infty}(-1)^{L}(2 L+1) \times \\
& C_{l 0 L 0}^{l^{\prime} 0} C_{l m_{l} L 0}^{l^{\prime} m_{l}} \mathcal{K}_{L}(\kappa R)
\end{aligned}
$$

originate from the re-expansion [18] of $\mathcal{K}_{l}(\kappa \mid \mathbf{x}-$ $\mathbf{R} \mid) Y_{l m_{l}}\left(\mathbf{n}_{\mathbf{x}-\mathbf{R}}\right)$ into $Y_{l m_{l}}\left(\mathbf{n}_{\mathbf{x}}\right)$ with the Clebsch-Gordan coefficients $C_{l m_{l} L 0}^{l^{\prime} m_{l}}$.

In order to derive an equation for $C_{l m_{l}}^{( \pm)}$we cast the radial wave $R_{l m_{l}}$ given by Eq. (11) into the superposition

$$
R_{l m_{l}}(\kappa|\mathbf{x}|)=a_{l}(\kappa) h_{l}^{(1)}(i \kappa|\mathbf{x}|)+b_{l}(\kappa) h_{l}^{(2)}(i \kappa|\mathbf{x}|)
$$

of outgoing and incoming radial waves $h_{l}^{(1)}$ and $h_{l}^{(2)}$ with amplitudes

$$
a_{l}(\kappa)=-\frac{\pi i^{l}}{2} C_{l m_{l}}^{(+)}+\frac{\pi i^{l}}{2} \sum_{l^{\prime}=0}^{\infty} \mathfrak{A}_{l l^{\prime}}^{\left(m_{l}\right)}(\kappa R) C_{l^{\prime} m_{l}}^{(-)}
$$

and

$$
b_{l}(\kappa)=\frac{\pi i^{l}}{2} \sum_{l^{\prime}=0}^{\infty} \mathfrak{A}_{l l^{\prime}}^{\left(m_{l}\right)}(\kappa R) C_{l^{\prime} m_{l}}^{(-)}
$$

The spherical Bessel functions of the third kind $h_{l}^{(1)}$ and $h_{l}^{(2)}$ are determined [17] in terms of $\mathcal{K}_{l}$ and $\mathcal{I}_{l}$ as $\mathcal{K}_{l}(z)=$ $-\left(\pi i^{l} / 2\right) h_{l}^{(1)}(i z)$ and $\mathcal{I}_{l}(z)=\left[h_{l}^{(1)}(i z)+h_{l}^{(2)}(i z)\right] /\left(2 i^{l}\right)$.

Since the amplitudes $a_{l}$ and $b_{l}$ of the outgoing and incoming waves are coupled [16] by the $S$-matrix elements $S_{l}$ of the scattering potential $U$, that is

$$
a_{l}(\kappa)=S_{l}(i \kappa) b_{l}(\kappa),
$$

we arrive at

$$
C_{l m_{l}}^{(+)}+\left[S_{l}(i \kappa)-1\right] \sum_{l^{\prime}=0}^{\infty} \mathfrak{A}_{l l^{\prime}}^{\left(m_{l}\right)}(\kappa R) C_{l^{\prime} m_{l}}^{(-)}=0 .
$$


Similarly we obtain from the second potential well, centered at $\mathbf{r}=\frac{1}{2} \mathbf{R}$, the relation

$$
C_{l m_{l}}^{(-)}+\left[S_{l}(i \kappa)-1\right] \sum_{l^{\prime}=0}^{\infty}(-1)^{l+l^{\prime}} \mathfrak{A}_{l l^{\prime}}^{\left(m_{l}\right)}(\kappa R) C_{l^{\prime} m_{l}}^{(+)}=0 .
$$

Equations (17) and (18) constitute a system of linear algebraic equations for $C_{l m_{l}}^{( \pm)}$determining via Eq. (9) the waves $\Psi^{( \pm)}$. Its solution is nonzero only if the corresponding determinant vanishes which provides us with a transcendental equation for $\kappa=\kappa(R)$, and thus for the interaction potential $\mathcal{V}$ defined by Eq. (2). The coefficients of these equations are determined by the $S$-matrix elements of the interaction potential $U$ between the heavy and the light atoms.

Zero-range limit. In order to test our method, we first consider a zero-range potential, for which only $s$-wave scattering occurs and the $S$-matrix elements read [19]

$$
S_{l}(i \kappa)-1=\frac{2 \kappa}{1 / a_{0}-\kappa} \delta_{l, 0} .
$$

In this case, the system Eqs. (17) and (18) reduces to two algebraic equations for $C_{00}^{( \pm)}$and has non-trivial solutions only if

$$
\left[S_{0}(i \kappa)-1\right] \mathfrak{A}_{00}^{(0)}(\kappa R)= \pm 1,
$$

with $\mathfrak{A}_{00}^{(0)}(\kappa R) \equiv[1 /(2 \kappa R)] e^{-\kappa R}$ defined by Eq. (12). This condition translates into equation

$$
\frac{1}{\xi-\alpha_{0} \rho} e^{-\xi}= \pm 1
$$

for $\xi \equiv \kappa R$ with the parameters

$$
\alpha_{0} \equiv \frac{R_{0}}{a_{0}} \text { and } \rho \equiv \frac{R}{R_{0}},
$$

and coincides with the equation for the bound-state energy obtained in Refs. [10, 19] for the case of the zerorange potential.

In the case of a $s$-wave resonance, that is $\alpha_{0}=0$, Eq. (21) has a solution $\xi=\xi_{*} \approx 0.57$ only for the plus sign on the right-hand side, which translates into the familiar Efimov potential

$$
\mathcal{V}^{(0)} \equiv-\frac{\left[\hbar \kappa_{+}(R)\right]^{2}}{2 \mu}=-\frac{\hbar^{2}}{2 \mu} \frac{\xi_{*}^{2}}{R^{2}},
$$

decaying with the second power of $R$.

$P$-wave resonance. Next we focus on the low-energy limit, that is on $|E| \ll \hbar^{2} /\left(\mu R_{0}^{2}\right)$, or $\kappa R_{0} \ll 1$, where the $S$-matrix elements

$$
S_{l}(i \kappa)-1=\frac{2 i}{\cot \left[\delta_{l}(i \kappa)\right]-i}
$$

in the $l$-th partial wave are determined [16] by the scattering phases $\delta_{l}(i \kappa)$ following [20] from the effective-range expansion

$$
(i \kappa)^{2 l+1} \cot \left[\delta_{l}(i \kappa)\right] \cong-1 / a_{l}+\left(r_{l} / 2\right) \kappa^{2} .
$$

A resonance in the $l$-th partial wave is reached when the absolute value of the effective scattering length $\left|a_{l}\right| \gg$ $R_{0}^{2 l+1}$. The effective range $r_{l}$ for $l>0$ is positive and linked 16, 21, 22] to the width of the resonance. Moreover, for any short-range potential $r_{l}$ has a lower bound [21], that is $r_{l} \geq \tilde{\alpha}_{l} R_{0}^{1-2 l}$ with the positive constant $\tilde{\alpha}_{l}$ determined by $l$.

Recently it has been shown [14] that in the unitary limit, defined as $1 / a_{1}=0$ and $r_{1}=0$, any characteristic length scale disappears and the three-body problem could exhibit the Efimov effect. However, due to the natural lower bound [21] on $r_{1}$, this limit is unphysical and eliminates [15] the possibility of the Efimov effect in our system. We now take into account this lower bound on $r_{1}$ and show that the effective potential, and the corresponding energy spectrum are induced by and depend explicitly on $r_{1}$ [2].

For this purpose we consider the case of a resonant $p$ wave, that is a partial wave with $l=1$, and substitute the $S$-matrix elements given by Eqs. (24) and (25) into Eqs. (17) and (18). Since $S_{0}(i \kappa)-1 \sim S_{1}(i \kappa)-1 \sim \kappa R_{0}$ and $S_{l>1}(i \kappa)-1 \sim\left(\kappa R_{0}\right)^{2 l+1}$, we neglect the small terms with $l=2,3, \ldots$ and arrive at two separate systems of equations for each projection $m_{l}$ of the angular momentum.

For $m_{l}= \pm 1$, Eqs. (17) and (18) simplify to two equations for $C_{1, \pm 1}^{( \pm)}$and have a non-trivial solution only if

$$
\left[S_{1}(i \kappa)-1\right] \mathfrak{A}_{11}^{( \pm 1)}(\kappa R)= \pm 1 .
$$

Since $S_{1}(i \kappa)$ is given by Eqs. (24) and (25) and $\mathfrak{A}_{11}^{( \pm 1)}(\kappa R) \equiv-\frac{3}{2}\left[(1+\kappa R) /(\kappa R)^{3}\right] e^{-\kappa R}$, Eq. (26) for $\xi=\kappa^{(1)} R$ and distances $R \geq 2 R_{0}$ reads

$$
\frac{(1+\xi)}{\beta \rho \xi^{2}-\alpha_{1} \rho^{3}-\frac{1}{3} \xi^{3}} e^{-\xi}= \pm 1
$$

with the dimensionless parameters

$$
\alpha_{1} \equiv \frac{R_{0}^{3}}{3 a_{1}} \quad \text { and } \quad \beta \equiv \frac{r_{1} R_{0}}{6} .
$$

In the resonant case, that is $\alpha_{1}=0$, Eq. (27) has a solution only for the plus sign on the right-hand side. In the limit of $0<\xi \ll 1$, we find $\xi \cong(\beta \rho)^{-\frac{1}{2}}$ for $\rho \gg 1$, which translates into the potential

$$
\mathcal{V}^{(1, \pm 1)} \equiv-\frac{\left[\hbar \kappa_{+}^{(1)}(R ; 0)\right]^{2}}{2 \mu} \cong-\frac{\hbar^{2}}{2 \mu} \frac{6}{r_{1} R^{3}},
$$

which is independent of $R_{0}$.

The form of the potentials $\mathcal{V}_{ \pm}^{(1, \pm 1)}\left(R ; \alpha_{1}\right) \equiv$ $-\left[\hbar^{2} /\left(2 \mu R_{0}^{2}\right)\right]\left(\xi_{ \pm} / \rho\right)^{2}$ is determined by the solutions 
$\xi_{ \pm}\left(\rho ; \alpha_{1}\right)$ of Eq. (27) and depends on the sign of $a_{1}$, that is on the sign of $\alpha_{1}$, Eq. (28). Indeed, for $\alpha_{1}>0$, i.e., in the case of the weakly-bound $p$ wave state in $U, \mathcal{V}_{+}^{(1, \pm 1)}$ as well as $\mathcal{V}_{-}^{(1, \pm 1)}$ approach for large distances, $R>R_{0}\left|\alpha_{1}\right|^{-1 / 3}$, the bound state energy $\varepsilon_{1} \equiv-\left(\alpha_{1} / \beta\right)$ of the light particle. For short distances, $R<R_{0}\left|\alpha_{1}\right|^{-1 / 3}, \mathcal{V}_{+}^{(1, \pm 1)}\left(R ; \pm\left|\alpha_{1}\right|\right)$ approach $\mathcal{V}_{+}^{(1, \pm 1)}\left(R ; \alpha_{1}=0\right)=\mathcal{V}^{(1, \pm 1)}(R)$.

In the case of a $p$-wave resonance, the matrix element $S_{1}$ corresponding to the resonant channel is of the same order as $S_{0}$ for the non-resonant channel [16]. Therefore, for $m_{l}=0$ we have to take into account in Eqs. (17) and (18) both the $s$ - and $p$-waves, which gives rise to a system of four algebraic equations for $C_{0,0}^{( \pm)}$and $C_{1,0}^{( \pm)}$, leading us to the relation

$$
\begin{array}{r}
1-\left(S_{0}-1\right)\left(S_{1}-1\right)\left(\mathfrak{A}_{00}^{(0)} \mathfrak{A}_{11}^{(0)}-\mathfrak{A}_{01}^{(0)} \mathfrak{A}_{10}^{(0)}\right)= \\
\mp\left[\left(S_{0}-1\right) \mathfrak{A}_{00}^{(0)}-\left(S_{1}-1\right) \mathfrak{A}_{11}^{(0)}\right] .
\end{array}
$$

According to Eqs. (12), (24), (25) and (28), we obtain $\mathfrak{A}_{01}^{(0)} \equiv \mathfrak{A}_{10}^{(0)}=-\frac{\sqrt{3}}{2}\left[(1+\kappa R) /(\kappa R)^{2}\right] e^{-\kappa R}$ and $\mathfrak{A}_{11}^{(0)} \equiv$ $\frac{3}{2}\left[\left(\kappa^{2} R^{2}+2 \kappa R+2\right) /(\kappa R)^{3}\right] e^{-\kappa R}$, and Eq. (30) for $\xi=$ $\kappa^{(0)} R$ takes the form

$$
\begin{gathered}
1+\frac{e^{-2 \xi}}{\left(\xi-\alpha_{0} \rho\right)\left(\beta \rho \xi^{2}-\alpha_{1} \rho^{3}-\frac{1}{3} \xi^{3}\right)}= \\
\pm e^{-\xi}\left[\frac{\left(\xi^{2}+2 \xi+2\right)}{\beta \rho \xi^{2}-\alpha_{1} \rho^{3}-\frac{1}{3} \xi^{3}}+\frac{1}{\xi-\alpha_{0} \rho}\right] .
\end{gathered}
$$

In the resonant case, $\alpha_{1}=0$, we have $\alpha_{0} \sim 1$ and Eq. (31) has a solution only for the plus sign on the righthand side. In the limit of $0<\xi \ll 1$, we find $\xi \cong$ $[2 /(\beta \rho)]^{\frac{1}{2}}$ for $\rho \gg 1$, giving rise to the potential

$$
\mathcal{V}^{(1,0)} \equiv-\frac{\left[\hbar \kappa_{+}^{(0)}(R ; 0)\right]^{2}}{2 \mu} \cong-\frac{\hbar^{2}}{\mu} \frac{6}{r_{1} R^{3}} .
$$

The potentials $\mathcal{V}_{ \pm}^{(1,0)}\left(R ; \alpha_{1}\right) \equiv-\left[\hbar^{2} /\left(2 \mu R_{0}^{2}\right)\right]\left(\xi_{ \pm} / \rho\right)^{2}$ determined by the solutions $\xi_{ \pm}\left(\rho ; \alpha_{1}\right)$ of Eq. (31) at $\alpha_{0} \sim 1$ and $\beta \sim 1$ are similar to $\mathcal{V}_{ \pm}^{(1, \pm 1)}$ with two qualitative differences: (i) the ranges of $\mathcal{V}_{ \pm}^{(1, \pm 1)}$ and $\mathcal{V}_{ \pm}^{(1,0)}$ are different and equal to $R_{1} \equiv R_{0}\left|\alpha_{1}\right|^{-1 / 3}$ and $R_{2} \equiv R_{0}\left(0.5\left|\alpha_{1}\right|\right)^{-1 / 3}$, respectively, and (ii) in the case of exact resonance, $\mathcal{V}^{(1,0)}$ given by Eq. (32) has the same asymptotic behavior as $\mathcal{V}^{(1, \pm 1)}$ defined by Eq. (29) with twice the amplitude.

Spectrum of induced $1 / R^{3}$-potential. Finally we focus on the dynamics of the two heavy particles dictated by the Schrödinger equation (3) with the potential $\mathcal{V}$ given by Eqs. (29) and (32) and induced by the $p$-wave resonance in the light-heavy interaction [2]. We emphasize that $\mathcal{V}$ is only meaningful for $R \gg R_{0}$, since for $R \sim R_{0}$ the dynamics is determined by the direct short-range forces.
The energies $E_{n}$ of the bound states with zero angular orbital momentum follow from the familiar WKB quantization rule [16]

$$
n-n_{0}=\frac{1}{\pi \hbar} \int_{R_{0}}^{R_{E_{n}}} \sqrt{M\left[E_{n}-\mathcal{V}(R)\right]} d R,
$$

giving rise 23, 24] to the spectrum

$$
E_{n}=-\frac{\hbar^{2}}{M R_{*}^{2}}\left(\frac{n_{0}-n}{g}\right)^{6}
$$

for the weakly bound states, induced exclusively by $1 / R^{3}$ potential. Here we have introduced the characteristic range

$$
R_{*} \equiv\left(2-\left|m_{l}\right|\right) \frac{3 M}{\mu r_{1}}
$$

of the effective potential $\mathcal{V}$ in the resonant case, that is $\alpha_{1}=0$, and $\left[n_{0}\right]-n=1,2, .$. , where the integer part $\left[n_{0}\right]$, determined by the phase of the wave function at the short distances $R \sim R_{0}$, plays a role of a three-body parameter and $g \equiv \Gamma\left(\frac{5}{6}\right) /\left[\sqrt{\pi} \Gamma\left(\frac{4}{3}\right)\right]$.

Since $\mathcal{V}$ defined by Eqs. (29) and (32) has a tail falling off faster than $-1 / R^{2}$, it supports [16] only a finite number $N_{0}$ of bound states with zero angular orbital moment. Indeed, $N_{0}$ can be estimated [16] by the WKB method and yields

$$
N_{0}=\frac{1}{\pi} \int_{R_{0}}^{\infty} \sqrt{\frac{R_{*}}{R^{3}}} d R=\frac{2}{\pi} \sqrt{\frac{R_{*}}{R_{0}}}=\frac{2}{\pi}\left[\frac{\left(2-\left|m_{l}\right|\right)}{r_{1} R_{0}} \frac{3 M}{m}\right]^{\frac{1}{2}},
$$

that is $N_{0}$ is determined by the square root of the ratio of the mass-ratio $M / m$ to the dimensionless effective range $r_{1} R_{0}$ of the $p$-wave resonance.

The appearance of $\mathcal{V}$ given by Eqs. (29) and (32) can be verified experimentally by scattering a heavy atom off the diatomic molecule consisting of a heavy and a light atom. The predicted three-body bound states manifest themselves as resonances in the cross-section of the atom-molecule scattering when we tune the magnetic field close to the $p$-wave Feshbach resonance. Moreover, due to the inverse-cube tail the cross-section $\sigma_{L}$ of the $L$-th partial wave has the unique behavior [25], $\sigma_{0}(E)=\pi R_{*}^{2} \ln ^{2}\left(M R_{*}^{2} E / \hbar^{2}\right)$ and $\sigma_{L>0}(E)=\pi R_{*}^{2}[(2 L+$ 1) $\left./\left(L^{2}+L\right)^{2}\right]$ at the low incident energy $E \ll \hbar^{2} /\left(M R_{*}^{2}\right)$. Summary and outlook. We have found a novel series of bound states in the three-body system consisting of a light particle and two heavy ones when the heavy-light short-range interaction potential has the $p$-wave resonance. In the case of an exact resonance, the effective potential is attractive and of long-range. Moreover, the spectrum of bound states is determined by the mass ratio of the heavy and light particles as well as the parameters of the heavy-light short-range potential. 
Our treatment is based on the Born-Oppenheimer approximation, which for the Efimov case has already been shown to provide the correct effective interaction potential [26] and is not limited [27] to large mass ratios. Therefore, we are confident that our approach is also adequate for the three-body system with a $p$-wave resonant interaction.

Acknowledgments. We are deeply indebted to F. Ferlaino, K. Fossez, R. Kaiser, D. Petrov, M. Ploszajczak, and R. Walser for stimulating discussions. We also appreciate the financial support by the German Science Foundation (DFG) in the framework of the SFB/TRR-21. M.A.E. and M.Y.I. are grateful to the Alexander von Humboldt Stiftung and M.A.E. thanks the Russian Foundation for Basic Research (grant 10-02-00914-a).

[1] V. Efimov, Phys. Lett. B 33, 563 (1970); Sov. J. Nucl. Phys. 12, 589 (1971); Nucl. Phys. A 210, 157 (1973)

[2] After our letter was submitted an article appeared by S. Zhu and S. Tan, Phys. Rev. A 87, 063629 (2013), which applies our method to derive the effective potentials for the arbitrary angular momentum resonances. It refers to an earlier arXiv version of the present Letter, arXiv:1303.5939v1.

[3] A.S. Jensen et al., Rev. Mod. Phys. 76, 215 (2004)

[4] E. Nielsen et al., Phys. Rep. 347, 373 (2001)

[5] R.E. Grisenti et al., Phys. Rev. Lett. 85, 2284 (2000); R. Brühl et al., Phys. Rev. Lett. 95, 063002 (2005)

[6] C. Chin et al., Rev. Mod. Phys. 82, 1225 (2010)

[7] F. Ferlaino and R. Grimm, Physics 3, 9 (2010)

[8] G. Barontini et al., Phys. Rev. Lett. 103, 043201 (2009); Phys. Rev. Lett. 104, 059901(E) (2010)

[9] E. Braaten and H.-W. Hammer, Phys. Rep. 428, 259 (2006); Annals Phys. 322, 120 (2007)

[10] A.C. Fonseca, E.F. Redish, and P.E. Shanley, Nuclear Physics A 320, 273 (1979)

[11] M.A. Efremov et al., Phys. Rev. A 80, 022714 (2009)
[12] F. Ferlaino et al., Phys. Rev. A 73, 040702(R) (2006)

[13] B. Deh et al., Phys. Rev. A 77, 010701(R) (2008); C. Marzok et al., Phys. Rev. A 79, 012717 (2009)

[14] E. Braaten et al., Phys. Rev. A 86, 012711 (2012)

[15] Y. Nishida, Phys. Rev. A 86, 012710 (2012)

[16] L.D. Landau and E.M. Lifshitz, Quantum Mechanics (Pergamon Press, Oxford, 1977)

[17] Handbook of Mathematical Functions, edited by M. Abramowitz and I.A. Stegun (Dover, New York, 1972)

[18] D.A. Varshalovich, A.N. Moskalev, V.K. Khersonskii, Quantum Theory of Angular Momentum, Chap. 5 (World Scientific, Singapore, 1988)

[19] Yu. N. Demkov and V. I. Ostrovskii, Zero-range Potentials and their Applications in Atomic Physics (Plenum Press, New York, 1988)

[20] N. Mott and H.S.W. Massey, Theory of Atomic Collisions (Oxford University Press, Oxford, 1965), Ch. II

[21] F. Chevy et al., Phys. Rev A 71, 062710 (2005); L. Pricoupenko, Phys. Rev. A 73, 012701 (2006); M. JonaLasinio, L. Pricoupenko, and Y. Castin, Phys. Rev. A 77, 043611 (2008); P. Zhang, P. Naidon, and M. Ueda, Phys. Rev. A 82, 062712 (2010); H.-W. Hammer and D. Lee, Ann. Phys. 325, 2212 (2010)

[22] Experimentally, the resonant regime can be achieved using Feshbach resonances. In the vicinity of a resonance in the $l$-th partial wave, corresponding to the magnetic field $B \simeq B_{l}$, the effective scattering length $a_{l}(B) \propto$ $1 /\left(B_{l}-B\right)$ and the range $r_{l}(B)$ is almost constant and determined by the width of the Feshbach resonance. Indeed, the cases of a broad or a narrow resonance correspond to $r_{1} R_{0} \sim 1$ or $r_{1} R_{0} \gg 1$, respectively.

[23] R.J. LeRoy and R.B. Bernstein, J. Chem. Phys. 52, 3869 (1970)

[24] For a more rigorous treatment we refer to T.O. Müller and H. Friedrich, Phys. Rev. A 83, 022701 (2011) and P. Raab and H. Friedrich, Phys. Rev. A 78, 022707 (2008)

[25] R. Shakeshaft, J. Phys. B: At. Mol. Phys. 5, L115 (1972); B. Gao, Phys. Rev. A 59, 2778 (1999)

[26] Y. Hahn and B.G. Giraud, Eur. Phys. J. A 1, 383 (1998)

[27] K. Helfrich, H.-W. Hammer, and D.S. Petrov, Phys. Rev. A 81, 042715 (2010) 